\title{
Clustered Planarity: Small Clusters in Eulerian Graphs
}

\author{
Eva Jelínková ${ }^{1}$, Jan Kára ${ }^{2}$, Jan Kratochvíl ${ }^{1,2}$, Martin Pergel $^{1, \star}$, \\ Ondřej Suchý ${ }^{1}$, and Tomáš Vyskočil ${ }^{1}$ \\ ${ }^{1}$ Department of Applied Mathematics \\ ${ }^{2}$ Institute for Theoretical Computer Science ${ }^{\star \star}$ \\ Charles University \\ Malostranské nám. 25, 11800 Praha, Czech Republic \\ \{eva, kara, honza, perm, suchy, whisky\}@kam.mff.cuni.cz
}

\begin{abstract}
We present several polynomial-time algorithms for c-planarity testing for clustered graphs with clusters of size at most three. The most general result concerns a special class of Eulerian graphs, namely graphs obtained from a fixed-size 3-connected graph by multiplying and then subdividing edges. We further give algorithms for 3-connected graphs, and for graphs with small faces. The last result applies with no restrictions on the cluster size.
\end{abstract}

\section{Introduction}

Clustered planarity (or shortly, c-planarity) has recently become an intensively studied topic in the area of graph and network visualization. In many situations one needs to visualize a complicated inner structure of graphs and networks. Clustered graphs - graphs with recursive clustering structures over the vertices - provide a possible model of such a visualization, and as such they find applications in many practical problems, e.g., management information systems, social networks or VLSI design tools [4. However, from the theoretical point of view, the computational complexity of deciding c-planarity is still an open problem and it is regarded as one of the challenges of the contemporary graph drawing. Our aim is to add another pebble to the mosaic of known partial results on c-planarity by studying the case of small clusters.

Regarding the graph notations, we follow standard notation on finite loopless graphs. A graph is an ordered pair $G=(V, E)$. By $\bar{G}$ we denote its edge complement (i.e., $\left.\left(V,\left(\begin{array}{c}V \\ 2\end{array}\right) \backslash E\right)\right)$. For a vertex $v \in V$ by $N(v)$ we denote its set of neighbors.

Let $G=(V, E)$ be a graph. A cluster set on $G$ is a set $\mathcal{C} \subseteq \mathcal{P}(V(G))$ such that for all $C, D \in \mathcal{C}$, either $C$ and $D$ are disjoint or they are in inclusion. The elements of $\mathcal{C}$ are called clusters. A clustered planar embedding of $(G, \mathcal{C})$ is a

\footnotetext{
* Supported by the grant GAUK 154907.

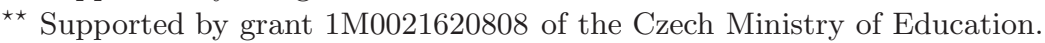


planar embedding $e m b$ of $G$ together with a mapping $e m b_{c}$ that assigns to every cluster $C \in \mathcal{C}$ a planar region $e m b_{c}(C)$ whose boundary is a closed Jordan curve and such that

- for each vertex $v \in V$ and every cluster $C \in \mathcal{C}$, it holds that $\operatorname{emb}(v) \in$ $e m b_{c}(C)$ if and only if $v \in C$,

- for every two clusters $C$ and $D$, the regions $e m b_{c}(C)$ and $e m b_{c}(D)$ are disjoint (in inclusion) if and only if $C$ and $D$ are disjoint (in inclusion, respectively), and

- for every edge $e \in E$ and every cluster $C \in \mathcal{C}$, the curve $e m b(e)$ crosses the boundary of $e m b_{c}(C)$ at most once.

The pair $(G, \mathcal{C})$ is called clustered planar (shortly c-planar) if it allows a clustered planar embedding.

It is well known that planar graphs can be recognized in polynomial (even linear) time. For c-planarity determining the time-complexity of the decision problem remains open; only partial results are known. For connected cluster graphs (i.e., when all clusters induce connected subgraphs), the problem can be solved in linear time 3. This work was extended to "almost" connected clustered graphs in 56] by designing an $O\left(n^{2}\right)$-time algorithm. Another important step was achieved by characterization of completely connected clustered graphs (where each cluster and its complement induce connected subgraphs): A completely connected clustered graph is c-planar if and only if the underlying graph is planar [1]. Another polynomially solvable case was identified in 2] (nested triples of clusters).

We propose to study the situation when all clusters are small, which means size at most 3. In Section 2 we first remind the notion of saturators and study its meaning in the case of small clusters. As the first observation we prove that c-planarity of vertex-3-connected graphs is solvable in polynomial time (small clusters assumed). Our most general result is a polynomial time algorithm for c-planarity of Eulerian graphs that can be obtained from vertex-3-connected graphs of fixed size by cloning and subdividing edges. This algorithm is mentioned in Section 2. As can be expected, the cornerstone of this algorithm is understanding the clustered planarity in the case of a single cycle as the underlying graph, since this sheds light on particular faces of the input graph. Though at first sight this case might sound trivial, it turns out far from being obvious. Our algorithm and further useful observations are presented in Section 3. The unexpected complexity of a single cycle was also encountered by di Battista et al. in [2] when solving c-planarity for small number (3) of clusters. Our last result concerns the case of small faces rather than small clusters. In Section 5 we present a polynomial algorithm for deciding c-planarity of graphs with fixed embeddings with all faces of size at most 4 . This result applies to clusters of all sizes, but only of a flat structure (i.e., when all clusters are on the same level, none being in inclusion with any other). 


\section{Saturators of Small Clusters}

Cortese et al. introduced the following notion in 2. A set $F$ is a saturator of $(G, \mathcal{C})$ if $F \subseteq E(\bar{G})$ and for each cluster $C \in \mathcal{C}$, the vertices of $C$ induce a connected subgraph in $G^{F}=(V(G), E(G) \cup F)$. The saturator $F$ is called planar if $G^{F}$ is planar. Note that the notion of planar saturators is slightly different than in 2]. The role of saturators is described by the following observation (stated in [2] in an equivalent formulation).

Lemma 1. The pair $(G, \mathcal{C})$ is c-planar if and only if there exists a saturator $F=F(G, \mathcal{C})$ such that $\left(G^{F}, \mathcal{C}\right)$ is c-planar.

For a cluster $A \in \mathcal{C}$, we call every pair of its vertices a cluster edge. We say that a cluster edge $e$ is present in a saturator $F$ if $e$ is an element of $F$. When it is clear from the context which saturator is considered, we omit its name and speak about present cluster edges only.

We further explore the meaning of saturators in certain special cases of graphs $G$ and cluster sets $\mathcal{C}$. In Section 3, we employ this idea in reducing a special case of c-planarity to the existence of a planar saturator of $(G, \mathcal{C})$, and then further to the bipartiteness and triangle-freeness of certain auxiliary graphs.

The following corollary of Lemma 1 is a first step in reducing c-planarity to the existence of a planar saturator. It states that the existence of a planar saturator is sufficient for clusters that do not induce a cycle.

Corollary 1. The pair $(G, \mathcal{C})$ is c-planar if and only if there exists a saturator $F$ such that $\left(G^{F}, \mathcal{C}^{\prime}\right)$ is c-planar, where $\mathcal{C}^{\prime}=\left\{C \in \mathcal{C}: G^{F}[C]\right.$ contains a cycle $\}$.

In this paper we mostly consider clusters of size at most three. We use the fact that if clusters are small, then there are only few possibilities of choosing present cluster edges in a saturator so that each cluster becomes connected.

A highly connected graph imposes other limitations on present cluster edges. Namely, in a fixed planar embedding of a 3-connected graph, each cluster edge can be drawn in at most one way. If we restrict ourselves both to 3-connected graphs and to clusters of size at most three, then it is possible to test c-planarity effectively. The c-planarity instance is transformed to a 2-SAT formula, where a variable represents the presence of a cluster edge, and a clause expresses a crossing.

Proposition 1. Let $G$ be a 3-connected graph and $\mathcal{C}$ a cluster set containing only clusters of size at most three. Then the c-planarity of $(G, \mathcal{C})$ can be decided in time $O\left(|\mathcal{C}|^{2} \cdot|G|\right)$.

\section{Three-Clusters on a Cycle}

Definition 1. Let $G$ be a cycle and $\mathcal{C}$ a set of at most three-element clusters on $V(G)$. We say that two cluster edges $\left\{a_{1}, a_{2}\right\}$ and $\left\{b_{1}, b_{2}\right\}$ conflict if the cyclic order of their vertices is abab. 
We say that two three-vertex clusters $A$ and $B \in \mathcal{C}$

- intersect if the cyclic order of their vertices along $G$ is aabbab,

- alternate if the cyclic order of their vertices along $G$ is ababab.

Given two clusters $A$ and $B$ we say that the vertices $a_{i} \in A$ and $b_{j} \in B$ are consecutive if there exists a path in $G$ from $a_{i}$ to $b_{j}$ that uses no other vertices of $A$ or $B$.

Lemma 2. If $G$ is a cycle and $\mathcal{C}$ contains only clusters of size at most three, then $(G, \mathcal{C})$ is c-planar if and only if there exists a planar saturator $F$.

The proof is a straightforward case analysis. It is omitted.

\subsection{Construction of Auxiliary Graphs $G_{1}, G_{1}^{M}$, and $G_{2}$}

We are given a pair $(G, \mathcal{C})$, where $G$ is a cycle and $\mathcal{C}$ contains only clusters of size at most three. According to Lemma 2, deciding the c-planarity of $(G, \mathcal{C})$ amounts to finding a planar saturator $F$. Thus, we need to pick suitable cluster edges for $F$ so that the graph $G^{F}$ makes every cluster connected and has a planar embedding.

Since $G$ is a cycle, we only distinguish two ways of drawing a cluster edge in a planar embedding: inside or outside the cycle $G$.

Conflicts of cluster edges impose restrictions on their embedding. For twovertex clusters, the situation is evident: each cluster edge must be drawn on one side of the cycle and any conflicting cluster edge must be drawn on the other side.

For three-vertex clusters the situation is more complicated, because we do not know in advance which cluster edges are present in the sought saturator and which are not. However, since $F$ is a saturator, we know that every cluster $C$ is connected in $G^{F}$. Hence, out of every pair of cluster edges of $C$, at least one is present in $F$. Thus, we consider pairs of three-vertex-cluster edges; these become vertices of an auxiliary graph $G_{1}$. Edges of two-vertex clusters will become vertices of $G_{1}$ also.

The formal construction of $G_{1}$ can be found below. There we also formalize the correspondence of vertices of $G_{1}$ and (pairs of) cluster edges. Here, for convenience, we use the notion of correspondence in an intuitive way.

For some vertex pairs $x$ and $y$ of $G_{1}$ the following holds: if any cluster edge corresponding to $x$ is present, then any present cluster edge corresponding to $y$ must be drawn on the other side of the cycle. Otherwise, a crossing would occur. Figure 1 illustrates some of those cases. We represent such a case by the edge between $x$ and $y$ in $G_{1}$.

We observe that if a vertex $x$ is non-isolated in $G_{1}$, then all present cluster edges corresponding to $x$ must be drawn on a common side of the cycle. For adjacent vertices the sides are distinct. Hence, if a bipartition of $G_{1}$ exists, it determines the drawing of all present cluster edges corresponding to non-isolated vertices (up to the choice of the inner and outer face of the cycle). Isolated vertices in $G_{1}$ are exceptional and we will not consider them to belong to any bipartity of $G_{1}$, because their corresponding cluster edges have, in a sense, more freedom. 

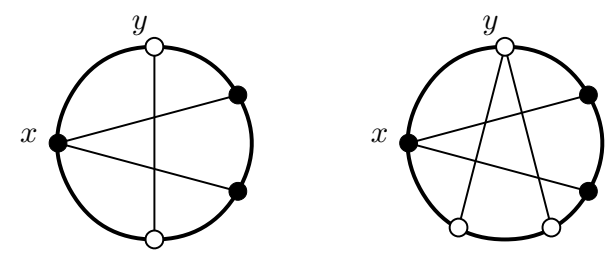

Fig. 1. Some of the situations when any cluster edge corresponding to $x$ must be drawn on the other side of the cycle than any cluster edge corresponding to $y$

The graph $G_{1}$ does not capture well the restrictions caused by alternating clusters - there may be several pairwise-alternating clusters that do not give rise to any edge of $G_{1}$. Hence, we define another auxiliary graph $G_{2}$. The vertices of $G_{2}$ are three-vertex clusters, and edges $\{A, B\}$ of $G_{2}$ express that clusters $A$ and $B$ alternate. We later prove that, for c-planarity, there may be no triangle in $G_{2}$.

In some cases there are vertices of $G_{1}$ whose corresponding cluster edges "behave in the same way" in any planar drawing of a saturator: either all present edges are drawn outside, or all inside, or all may be drawn on both sides. In the bipartition language, such vertices must either belong to a common bipartity of $G_{1}$ in any bipartition, or they must be all isolated. We need to "unify" them. Hence, we create the graph $G_{1}^{M}$ from $G_{1}$ by repeated merging of certain vertex tuples into groups.

The formal construction of the graphs $G_{1}, G_{2}$ and $G_{1}^{M}$ follows.

\section{Algorithm: Creation of $G_{1}$}

Input: $G=(V, E)$, a set $\mathcal{C}$ of clusters

Output: the graph $G_{1}$

$V\left(G_{1}\right):=\left\{x_{A, v}: A \in \mathcal{C},|A|=3, v \in A\right\} \cup\left\{x_{A}: A \in \mathcal{C},|A|=2\right\}$

$E\left(G_{1}\right):=\emptyset$

1. For every two clusters $A=\left\{a_{1}, a_{2}\right\}$ and $B=\left\{b_{1}, b_{2}\right\}$ whose vertices have the cyclic order $a_{1}, b_{1}, a_{2}, b_{2}$, set $E\left(G_{1}\right)=E\left(G_{1}\right) \cup\left\{\left\{x_{A}, x_{B}\right\}\right\}$.

2. For every two clusters $A=\left\{a_{1}, a_{2}\right\}$ and $B=\left\{b_{1}, b_{2}, b_{3}\right\}$ whose vertices have the cyclic order $a_{1}, b_{1}, a_{2}, b_{2}, b_{3}$, set $E\left(G_{1}\right)=E\left(G_{1}\right) \cup\left\{\left\{x_{A}, x_{B, b_{1}}\right\}\right\}$.

3. For every two clusters $A=\left\{a_{1}, a_{2}, a_{3}\right\}$ and $B=\left\{b_{1}, b_{2}, b_{3}\right\}$ whose vertices have the cyclic order $a_{1} a_{2} b_{1} b_{2} a_{3} b_{3}$, set $E\left(G_{1}\right):=E\left(G_{1}\right) \cup\left\{\left\{x_{A, a_{3}}, x_{B, b_{3}}\right\}\right\}$.

4. For every two alternating clusters $A$ and $B$ such that the vertices $y_{A}$ and $y_{B}$ both have degree exactly one in $G_{2}$, and for every pair of their vertices $a_{i} \in A$ and $b_{j} \in B$ that are consecutive and both non-isolated in $G_{1}$, set $E\left(G_{1}\right):=E\left(G_{1}\right) \cup\left\{\left\{x_{A, a_{i}}, x_{B, b_{j}}\right\}\right\}$.

$$
\begin{gathered}
V\left(G_{2}\right):=\left\{y_{A}: A \in \mathcal{C},|A|=3\right\} \\
E\left(G_{2}\right):=\left\{\left\{y_{A}, y_{B}\right\}: A \text { and } B \text { alternate }\right\} .
\end{gathered}
$$



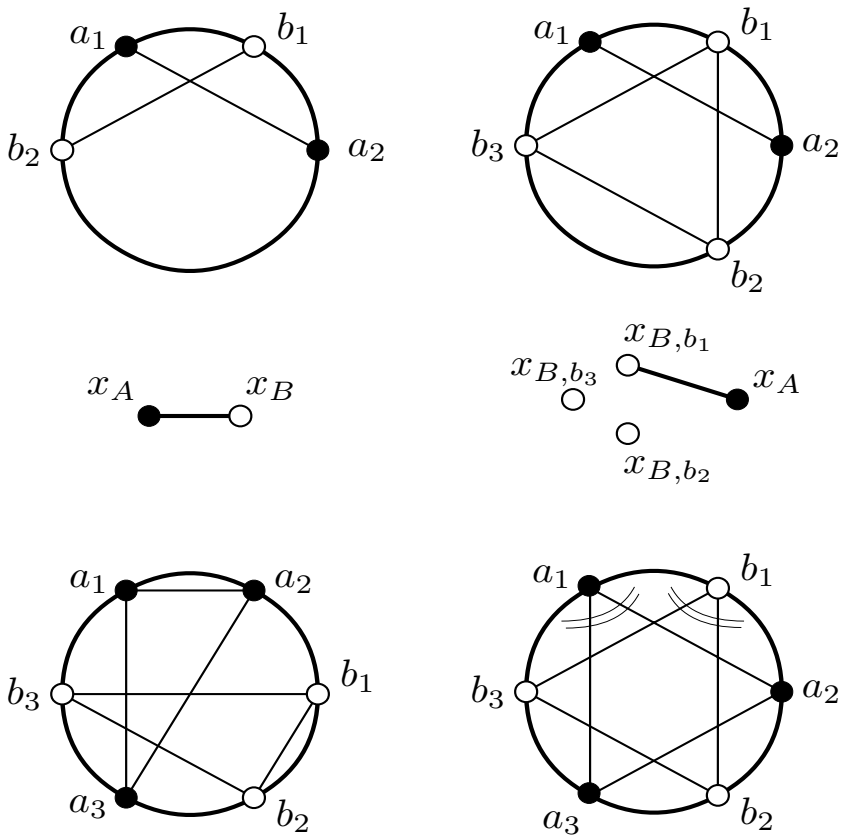

$$
\begin{array}{ll}
x_{A, a_{1}} & \bullet^{x_{A, a_{2}}} \\
x_{B, b_{3}} & \bigcirc^{x_{B, b_{2}}} \\
x_{B, b_{1}}
\end{array}
$$

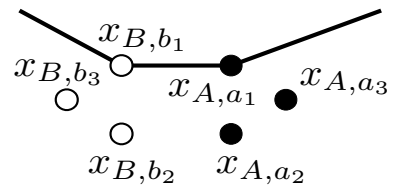

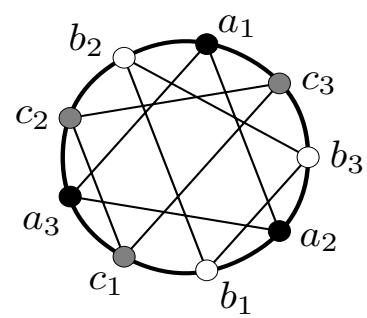

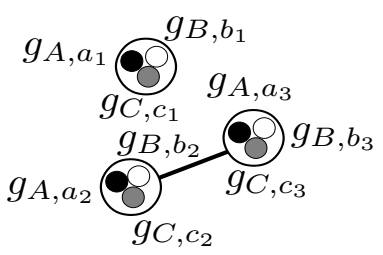

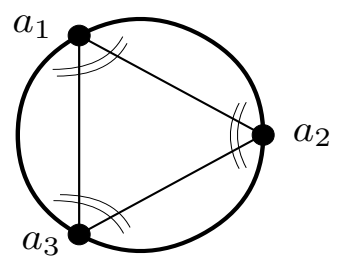
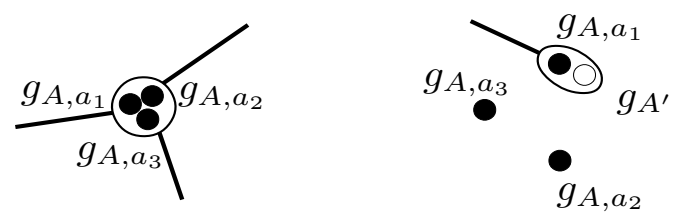

Fig. 2. Illustration of rules 120 , 4 目 and 7 
To formalize the correspondence of clusters and cluster edges with vertices of $G_{1}$ and $G_{2}$, we introduce the following definition.

Definition 2. We say that a cluster edge $e=\left\{a_{i}, a_{j}\right\}$ of a cluster $A$ in $G$ corresponds to a vertex $v$ of $G_{1}$ if $v=x_{A, a_{i}}$ or $v=x_{A, a_{j}}$, or if $v=x_{A}$.

We say that a cluster $B$ corresponds to a vertex $u$ of $G_{2}$ if $u=y_{B}$.

The following algorithm creates the graph $G_{1}^{M}$ from $G_{1}$ together with a mapping $g: V\left(G_{1}\right) \rightarrow V\left(G_{1}^{M}\right)$. The vertices of $G_{1}^{M}$ will represent groups of vertices of $G_{1}$ and the mapping $g$ will assign to each vertex the group to which it belongs. For short, we write $g_{A, a_{i}}$ or $g_{A}$ instead of $g\left(x_{A, a_{i}}\right)$ or $g\left(x_{A}\right)$, respectively.

The algorithm starts with one-vertex groups equal to vertices of $G_{1}$ and then merges certain groups using the following procedure.

\section{Procedure: Merge}

Input: vertex groups $g_{1}, g_{2}, \ldots, g_{k} \in V\left(G_{1}^{M}\right)$

Output: modifies $G_{1}^{M}$

- Replace the groups $g_{1}, g_{2}, \ldots, g_{k}$ with a newly created vertex group $w$, and set the edges in $G_{1}^{M}$ so that

$$
N(w)=N\left(g_{1}\right) \cup N\left(g_{2}\right) \cup \cdots \cup N\left(g_{k}\right) \backslash\left\{g_{1}, g_{2}, \ldots, g_{k}\right\} .
$$

- If there were two indices $1 \leq i, j \leq k$ (not necessarily distinct) such that $g_{i}$ and $g_{j}$ were adjacent then add a loop $\{w, w\}$.

- For all vertices $v$ in $g^{-1}\left(g_{1} \cup g_{2} \cup \cdots \cup g_{k}\right)$ set $g(v):=w$.

\section{Algorithm: Creation of $G_{1}^{M}$}

Input: the graph $G_{1}$

Output: the graph $G_{1}^{M}$, a mapping $g: V\left(G_{1}\right) \rightarrow V\left(G_{1}^{M}\right)$

$G_{1}^{M}:=G_{1}, g:=\mathrm{id}$

5. For each cluster $A=\left\{a_{1}, a_{2}, a_{3}\right\}$ which alternates with at least two other clusters $B=\left\{b_{1}, b_{2}, b_{3}\right\}$ and $C=\left\{c_{1}, c_{2}, c_{3}\right\}$ in the way $a_{1}, c_{3}, b_{3}, a_{2}, b_{1}$, $c_{1}, a_{3}, c_{2}, b_{2}$, do merge $\left(g_{A, a_{1}}, g_{B, b_{1}}, g_{C, c_{1}}\right)$, merge $\left(g_{A, a_{2}}, g_{B, b_{2}}, g_{C, c_{2}}\right)$, and $\operatorname{merge}\left(g_{A, a_{3}}, g_{B, b_{3}}, g_{C, c_{3}}\right)$.

6. For every three-vertex cluster $A$ having all corresponding vertices $g_{A, a_{i}}$ non-isolated in $G_{1}^{M}$, do merge $\left(g_{A, a_{1}}, g_{A, a_{2}}, g_{A, a_{3}}\right)$.

7. For every two clusters $A^{\prime}=\left\{a_{1}, a_{2}\right\}$ and $A=\left\{a_{1}, a_{2}, a_{3}\right\}$ such that $g_{A, a_{1}}$ is not isolated in $G_{1}^{M}$, do merge $\left(g_{A, a_{1}}, g_{A^{\prime}}\right)$.

Having created all the auxiliary graphs $G_{1}, G_{1}^{M}$ and $G_{2}$, it is easy to decide if the input pair $(G, \mathcal{C})$ is c-planar, as stated in Theorem 1. Before stating the theorem, we present several auxiliary lemmas.

Lemma 3. Let $F$ be a planar saturator. Then in $G^{F}$, the following is true: 
1. if there is an edge between the vertices $x$ and $y$ in $G_{1}$, then any present cluster edge corresponding to $x$ is drawn inside the cycle $G$, and any present cluster edge corresponding to $y$ is drawn outside the cycle $G$, or vice versa.

2. the present cluster edges corresponding to vertices in $g^{-1}(v)$ such that $v$ is non-isolated in $G_{1}^{M}$ are drawn either all inside or all outside the cycle $G$.

3. if there is an edge between the vertices $x$ and $y$ in $G_{1}^{M}$ then any present cluster edge corresponding to $g^{-1}(x)$ is drawn inside the cycle $G$, and any present cluster edge corresponding to $g^{-1}(y)$ is drawn outside the cycle $G$, or vice versa.

Proof. We follow the creation of $G_{1}$ and prove that the first part of the Lemma holds after every step. Before any rule is applied, there are no edges in $G_{1}$ and it holds trivially. Then a step according to rule 1, 2 or 3 adds one new edge, say, $x y$. For all these rules, it is not hard to see that if an edge corresponding to $x$ and an edge corresponding to $y$ are drawn on the same side of the cycle $G$, then they cross each other. Hence, after a step 1, 2, or 3. the first part remains valid.

Let us consider a step according to rule 4. We use the same notation as in the description of this step, so we have two clusters $A$ and $B$, and let $x=x_{A, a_{1}}$ and $y=x_{B, b_{1}}$. Note that by definition of rule 4 , the vertices $x$ and $y$ are already non-isolated. Thus all present cluster edges corresponding to $x$ must be drawn on the same side of the cycle, and the same holds for $y$.

Assume for contradiction that there are cluster edges corresponding to $x$ and $y$ both inside the cycle $G$. Then it must be edges $a_{1} a_{3}$ and $b_{1} b_{2}$, because they are the only pair without a crossing. By the above argument, the edge $a_{1} a_{2}$ can only be drawn on the same side as $a_{1} a_{3}$; but that is not possible because of $b_{1} b_{2}$. So $a_{1} a_{2}$ is not present. Then $a_{2} a_{3}$ is present and drawn outside. Similarly, $b_{1} b_{3}$ is not present, and there is no way to draw $b_{2} b_{3}-$ a contradiction.

The second part is proved by a straightforward case analysis of the algorithm steps. The third part is an easy consequence of the first two.

Lemma 4. If $(G, \mathcal{C})$ contains three pairwise alternating clusters, then $(G, \mathcal{C})$ is not c-planar.

Proof. Let $A, B$, and $C$ be the three clusters, and assume for contradiction that $(G, \mathcal{C})$ is c-planar. We use Lemma 2 to do a straightforward case analysis of the cluster edges present in a planar drawing. If present cluster edges of $A$ are all drawn on the same side of the cycle, then present cluster edges of $B$ must be drawn on the other side, and there is no way to draw at least two cluster edges of $C$ without crossing. The other possibility is that $A$ has one cluster edge drawn inside and one outside the cycle. Then the same holds for $B$, and again, there is no way to draw $C$.

Lemma 5. If $G$ has a planar saturator, then $G_{1}^{M}$ is bipartite and $G_{2}$ is trianglefree.

Proof. First assume that $G_{1}^{M}$ is not bipartite. Then it contains an odd cycle $C$, $V(C)=\left\{v_{1}, v_{2}, v_{3} \ldots v_{2 k+1}\right\}, 0 \leq k$. Without loss of generality we can assume that 
cluster edges corresponding to vertices in $g^{-1}\left(v_{1}\right)$ are drawn inside the cycle. By Lemma 3 we know that $g^{-1}\left(v_{2}\right)$ is outside, by the same argument $g^{-1}\left(v_{3}\right)$ is inside etc. But then both $g^{-1}\left(v_{1}\right)$ and $g^{-1}\left(v_{2 k+1}\right)$ are drawn inside, which is not possible again by Lemma 3], a contradiction.

If $G_{2}$ contains a triangle, then there are three pairwise alternating clusters and the instance is not c-planar by Lemma 4.

Lemma 6. If $G_{2}$ is triangle-free and $G_{1}^{M}$ is bipartite, then $(G, \mathcal{C})$ has a planar saturator $F$.

Proof. Let $I$ be the set of isolated vertices of $G_{1}^{M}$. Let us fix a drawing of the cycle $G$ into the plane and some bipartition of $G_{1}^{M} \backslash I$ for the rest of this section. As $G$ is a cycle, any its drawing has well-defined inner and outer face, so drawing an edge of $G^{F}$ inside or outside of the cycle is well defined. The idea behind our drawing is that edges represented by non-isolated vertices of $G_{1}^{M}$ in the first part are drawn inside the cycle and edges in the second part are drawn outside the cycle. Vertices of $I$ do not impose any restriction and therefore the edges represented by them can be drawn both inside or outside the cycle.

The rest of the proof, which is a long and technical case analysis, is omitted.

Theorem 1. Let $G$ be a cycle, let $\mathcal{C}$ contain only clusters of size at most three, and let $G_{1}^{M}$ and $G_{2}$ be the graphs constructed for $(G, \mathcal{C})$ using the algorithms above. Then the pair $(G, \mathcal{C})$ is c-planar if and only if $G_{1}^{M}$ is bipartite and $G_{2}$ is triangle-free.

Proof. By Lemma 2, a pair $(G, \mathcal{C})$ satisfying the assumptions is c-planar if and only if it has a planar saturator. Lemmas 5 and 6 provide the rest of the proof.

Corollary 2. Let $G$ be a cycle and let $\mathcal{C}$ contain only clusters of size at most three. Then the c-planarity of $(G, \mathcal{C})$ can be decided in time $O\left(|V(G)|+|\mathcal{C}|^{3}\right)$.

\section{Three-Clusters on Rib-Eulerian Graphs}

As a generalization of the previous section, in this section we mention the algorithm for c-planarity of a special subclass of Eulerian graphs. Let $k$ be a constant; we call a graph $k$-Rib-Eulerian if it is Eulerian, and if it can be obtained from a 3 -connected graph on $k$ vertices by multiplying some edges, and then subdividing some edges. Figure 3 gives an example of such a graph.

We say that a path whose inner vertices have degree two and the outer vertices have degree larger than two is a rib. Thus a $k$-Rib-Eulerian graph consists of $k$ vertices of degree at least four that are interconnected by ribs. A vertex of degree at least four is called a branching vertex. A cluster is called a branch cluster if it contains a branching vertex, and non-branch cluster otherwise.

We want to decide the c-planarity of a pair $(G, \mathcal{C})$, where $G$ is a Rib-Eulerian graph. First, we deal with the branch clusters. We try all the possibilities of choosing saturator edges to those clusters, we add each chosen edge to $G$ twice (in order not to break its Eulericity) and we run the rest of the algorithm for 


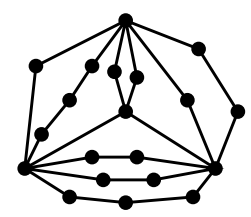

Fig. 3. Example of a Rib-Eulerian graph created from $K_{4}$

each of the posibilities. Clearly, the pair $(G, \mathcal{C})$ is c-planar if and only if it is c-planar for at least one of the saturator choices. Moreover there are constantly many choices to check, since there can only be $O(k)$ branch clusters in a $k$-RibEulerian graph and $k$ is a constant. Hence the rest of the algorithm sketched bellow only deals with non-branch clusters.

As a next step, we seek a suitable planar embedding of $G$. The planar embedding of the underlying 3-connected graph is unique (up to the choice of the outer face). Hence our main issue is to find the order of ribs originating from a common edge of the underlying graph. This is done with respect to clusters in $\mathcal{C}$, because they force adjacencies of certain ribs.

When a suitable planar embedding of $G$ is obtained, we utilize the algorithm of Section 3 that deals with cycles. In a planar embedding of a Rib-Eulerian graph, the boundary of each face is a cycle. All the restrictions for cluster edges on a cycle apply in this case as well. And more of them appear, because in this case, "the outside" of faces is more complex. Basically, we create the auxiliary graphs $G_{1}$ and $G_{1}^{M}$ for the whole graph at once, applying the rules from the previous section on (parts of) clusters lying in the same face. The graph $G_{2}$ is created for each face separately. We then reduce the c-planarity of $(G, \mathcal{C})$ to the bipartiteness of the graph $G_{1}^{M}$ and the triangle-freeness of the graphs $G_{2}$ for each face.

Due to space limitations, details of the algorithm and the proof of the correctness and running time are omitted. We just mention the summarizing theorem.

Theorem 2. The c-planarity of $(G, \mathcal{C})$ can be decided in time $O\left(3^{k} \cdot n^{3}\right)$ for $G$ being $k$-Rib-Eulerian with $n$ vertices and $\mathcal{C}$ containing clusters of size at most 3.

\section{Clustered Planarity on Graphs with Small Faces}

In this section, we show that the c-planarity problem can be solved in polynomial time for 3-connected graphs with faces of size at most 4 and with cluster sets where every two clusters are disjoint. Thus in this section we always assume the graph $G$ is 3-connected with faces of size at most 4 . First, we define the notion of extended graph of a cluster $A$. Informally, the extended graph contains all cluster edges that can be added to the drawing of $G$ and can help connecting $A$.

Definition 3. Let $G=(V, E)$ be a plane graph and $\mathcal{C}$ a cluster set. For a cluster $A \in \mathcal{C}$ we define the extended graph of $A$, called $G_{E}(A)$, on the set of vertices $A$. Two vertices $u, v$ in $G_{E}(A)$ are adjacent if and only if either $\{u, v\} \in E$ or $u$ and $v$ are in the same face of $G$ and in different connected components of $G[A]$. 


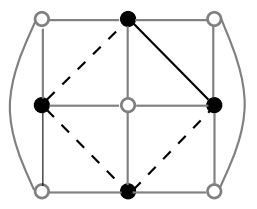

Fig. 4. The extended graph of a cluster $A$ in its unique embedding. Vertices of $A$ are depicted as solid black disks, other vertices of $G$ are depicted as grey circles. Edges of $G$ are drawn as solid (either grey or black) lines, edges of $G_{E}(A)$ are drawn as black lines (dashed in case the edge is not in $G$ ).

As seen in Figure 4, the drawing of $G$ defines drawings of extended graphs:

Lemma 7. Let $(G, \mathcal{C})$ be as above. Then for each $A \in \mathcal{C}$ the graph $G_{E}(A)$ is planar and its (unique) planar embedding is defined by the embedding of $G$.

The key idea of our approach is to examine the drawing of the extended graph $G_{E}(A)$ while forgetting about the edges of $G$ itself. We continue by presenting two lemmas about extended graphs.

Lemma 8. Let $G$ be a 3-connected graph with each face of size at most four, $\mathcal{C}$ be a cluster set, $A \in \mathcal{C}$ and $G_{E}(A)$ as above. Let $f$ be a face of $G_{E}(A), B \in \mathcal{C}$ and $B^{\prime}$ be the vertices of $B$ inside face $f$. Then for every planar saturator $F$ of $(G, \mathcal{C})$, the graph $G^{F}\left[B^{\prime}\right]$ is connected.

The following lemma uses the notion of labeled dual of $G_{E}(A)$. Let $\mathcal{C}$ be a cluster set such that every two clusters are disjoint. A labeled dual of $G_{E}(A)$, called $G_{E}^{d}(A)$, is a multigraph whose vertices are faces of $G_{E}(A)$. Edges of $G_{E}^{d}(A)$ are labeled by clusters of $\mathcal{C}$, and $G_{E}^{d}(A)$ contains an edge between $f$ and $f^{\prime}$ labeled by $B$ if $f, f^{\prime}$ are adjacent and cluster $B$ has vertices inside both these faces. See Figure 5 for an illustration of labeled dual.

Lemma 9. Let $G$ be as above, and $\mathcal{C}$ a cluster set such that every two clusters in $\mathcal{C}$ are disjoint. If $G_{E}^{d}(A)$ has a cycle whose edges have at least two different labels for some $A \in \mathcal{C}$, then $(G, \mathcal{C})$ is not c-planar.

Now we are ready to show our main theorem.

Theorem 3. The c-planarity of $(G, \mathcal{C})$, where $G$ is a 3-connected planar graph with faces of size at most 4 and $\mathcal{C}$ is a cluster set such that every two clusters are disjoint, can be decided in time $O\left(|V(G)|^{2}\right)$.

Proof. (sketch) The idea of the proof is as follows: As every 3-connected graph has a unique embedding into the plane (up to the choice of the outer face), we can assume $G$ has a fixed embedding. We pick arbitrary $A \in \mathcal{C}$. By Lemma 8 in each face $f$ of $G_{E}(A)$, each cluster has to be connected by any saturator. Thus in each face, we solve a small c-planarity problem. Then we connect parts of clusters in different faces greedily and by planar-duality argument we show that if there was no cycle using two labels in $G_{E}^{d}(A)$ (otherwise by $\operatorname{Lemma} 9(G, \mathcal{C})$ is not c-planar), the cluster $A$ can be still connected. 

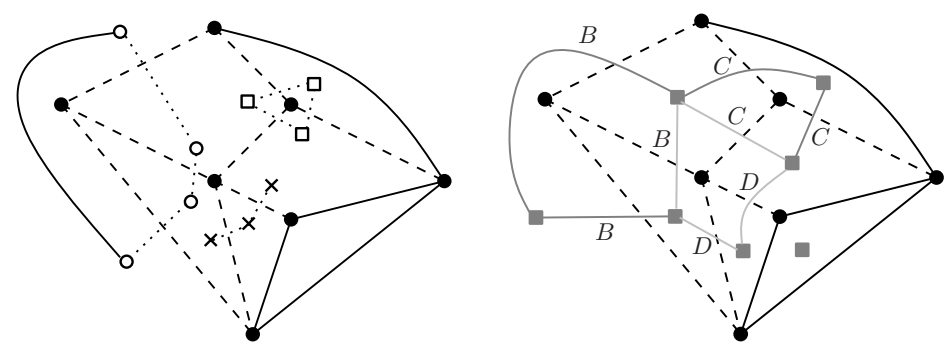

Fig. 5. Left: A graph $G_{E}(A)$ (discs) together with extended graphs of clusters $B, C$ and $D$ (circles, boxes, crosses). Right: A graph $G_{E}(A)$ (black) with its labeled dual $G_{E}^{d}(A)$ (grey) containing a cycle (light grey).

\section{Acknowledgement}

The authors would like to thank Tomáš Chudlarský for valuable discussions.

\section{References}

1. Cornelsen, S., Wagner, D.: Completely Connected Clustered Graphs. Journal of Discrete Algorithms 4(2), 313-323 (2006)

2. Cortese, P.F., Di Battista, G., Patrignani, M., Pizzonia, M.: Clustering Cycles into Cycles of Clusters. Journal of Graph Algorithms and Applications, Special Issue on the 2004 Symposium on Graph Drawing, GD 2004 9(3), 391-413 (2005)

3. Dahlhaus, E.: Linear time algorithm to recognize clustered planar graphs and its parallelization. In: Lucchesi, C.L., Moura, A.V. (eds.) LATIN 1998. LNCS, vol. 1380, pp. 239-248. Springer, Heidelberg (1998)

4. Feng, Q.W., Cohen, R.F., Eades, P.: Planarity for clustered graphs. In: Spirakis, P.G. (ed.) ESA 1995. LNCS, vol. 979, pp. 213-226. Springer, Heidelberg (1995)

5. Gutwenger, C., Jünger, M., Leipert, S., Mutzel, P., Percan, M., Weiskircher, R.: C-planarity testing of clustered graphs. In: Goodrich, M.T., Kobourov, S.G. (eds.) GD 2002. LNCS, vol. 2528, pp. 220-235. Springer, Heidelberg (2002)

6. Gutwenger, C., Jünger, M., Leipert, S., Mutzel, P., Percan, M., Weiskircher, R.: Subgraph induces planar connectivity augmentation. In: Bodlaender, H.L. (ed.) WG 2003. LNCS, vol. 2880, pp. 261-272. Springer, Heidelberg (2003) 